\title{
JOURNEY OF AFRICAN SWINE FEVER VIRUS IN EASTERN EU STATES \\ Stelian Baraitareanu ${ }^{1}$, Dragos Cobzariu ${ }^{2}$, Mihaela Popp ${ }^{3}$, Marius Valer Campeanu ${ }^{4}$, Doina Danes ${ }^{5}$
}

\begin{abstract}
:
INTRODUCTION: In 2007, African swine fever virus (ASFv) broken its well-known boundaries. This was the reference year for the first report of African swine fever (ASF) in Georgia. Subsequently, the virus reached pigs and boars in Armenia and Russia. From the Caucasus area, ASFv jumped in all directions, between the Black Sea and the Caspian Sea, in relation to the density of backyard pigs and their trade. In the next ten years there have been notifications and registrations of ASFv outbreaks in Russia, Azerbaijan, Ukraine, Belarus, Lithuania, Poland, Estonia, Latvia, Moldova and the Czech Republic. Romania faced the first ASFv outbreak at the end of July 2017, in backyard pigs.

OBJECTIVES: The aim of study is to analyse the ways ASFv spread from and into different regions recorded by Eastern European states.

METHODS: The immediate notifications on ASFv to the World Organisation for Animal Health (OIE) were analysed from the Eastern-European states between 2007 and 2017. The analysis took into consideration the first occurrence of the disease under scrutiny in the country and the follow-up reports, in relation with the geospatial distribution of the outbreaks.

RESULTS: The main route of ASFv introduction into local pig populations indicated by the Member States of the European Union was the trans-boundary circulation of boars. However, the spread of ASFv through both, wild and domestic pigs and also by the human alimentary customs/traditions in the affected areas shouldn't be ignored. Three cycles of ASFv transmission have been identified and described by the epidemiologists: the domestic cycle, the sylvatic cycle and the tick-pig cycle.

CONCLUSION: None of the ways to disseminate the ASFv should be excluded, and the origin of the first outbreaks remains unknown or inconclusive in Eastern EU states.
\end{abstract}

UDC Classification: 616.9; DOI: http://dx.doi.org/10.12955/cbup.v6.1262

Keywords: ASF; ASFv; epidemiology; mode of transmission.

\section{Introduction}

African swine fever (ASF) is one of the world's most dangerous viral diseases of swine, with serious social and economic impacts mainly for countries where pigs are an important source of food (SanchezVizcaino \& Arias, 2012). ASF outbreaks have been reported in several African, European and American countries (including the Caribbean), as well as in several regions of the Russian Federation, from the Palearctic regions to the Mongolian borders (Sanchez-Vizcaino \& Arias, 2012; Kolbasovet al, 2017; Sanchez-Cordon et al, 2018). However, ASF has been described as endemic only in sub-Saharan Africa, in the Island of Sardinia (Italy) and, recently, in parts of the Russian Federation (Gallardo et al, 2015). The endemic status of ASF in sub-Saharan Africa dates back to 1909 when the introduction of European domestic pigs in Kenya revealed the presence of the African swine fever virus (ASFv) (Montgomery, 1921). Between 1957 and 1995 outbreaks of ASF were reported in several non-African states but the disease has been eradicated in almost all contaminated regions; only one exception was recorded: the Island of Sardinia (Sanchez-Vizcaino \& Arias, 2012). In 2007, ASFv broken its well-known boundaries, and spread outside sub-Saharan Africa to the Trans-Caucasus region (Sanchez-Cordon et al, 2018).

This was the reference year for the first report of ASF in Georgia (Ramishvili, 2007). Subsequently, the virus reached pigs and boars throughout the Caucasus in Armenia, the Russian Federation (wild boars in the Republic of Chechnya) and Azerbaijan (Beltran-Alcrudo et al, 2008).

From the Caucasus area, ASFv jumped in all directions, between the Black Sea and the Caspian Sea, in relation with the density of backyard pigs and their trade. In the next ten years, outbreaks of ASF were announced in Russia, Azerbaijan, Ukraine, Belarus, Lithuania, Poland, Estonia, Latvia, Moldova and

\footnotetext{
${ }^{1}$ Stelian Baraitareanu, Faculty of Veterinary Medicine, University of Agronomic Science and Veterinary Medicine of Bucharest, Romania; stelianbaraitareanu@fmvb.ro

${ }^{2}$ Dragos Cobzariu, Faculty of Veterinary Medicine, University of Agronomic Science and Veterinary Medicine of Bucharest, Romania; doruvet@ gmail.com

${ }^{3}$ Mihaela Popp, Faculty of Veterinary Medicine, University of Agronomic Science and Veterinary Medicine of Bucharest, Romania; mikacristi@yahoo.com

${ }^{4}$ Marius Valer Campeanu, Faculty of Veterinary Medicine, University of Agronomic Science and Veterinary

Medicine of Bucharest, Romania; safticam@yahoo.com

${ }^{5}$ Doina Danes, Faculty of Veterinary Medicine, University of Agronomic Science and Veterinary Medicine of Bucharest, Romania; danes.doina@gmail.com
} 
the Czech Republic. Romania faced its first ASF outbreak at the end of July 2017, in backyard pigs (Grigore, 2017).

The aim of this study is to analyse the ways ASFv spreads from and into different regions recorded by the Eastern European states.

\section{Material and Methods}

The immediate notifications of the any new ASF outbreak to the World Organisation for Animal Health (OIE) coming from the Eastern-European states, between 2007 and 2017 were analysed. The analysis of the spread of ASFv, from and into different regions, covered the temporal and spatial patterns of ASF outbreaks and considered all cases, including domestic pigs and wild boar.

The analysis took into consideration the first occurrence of the disease under scrutiny in the country and the follow-up reports, in relation with the geospatial distribution of the outbreaks, for each year. Data from the World Animal Health Information Database Interface (WAHID, 2018) were extracted and collated using the spreadsheet software Microsoft Excel 2013 before carrying out the analyses. The WAHIS database contains the outbreak report of African swine fever including the date of the start of the event, outbreak status, date of the outbreak resolution, the epidemiological unit type, location, (latitude, longitude), description of affected population, and species and the number of affected animals (at risk, cases, deaths, killed and disposed of) (WAHID, 2018). Since the European Food Safety Authority (EFSA) provided in its recent scientific report on ASF epidemiology (EFSA et al, 2017) comprehensive epidemiological data including the evaluation of the risk factors contributing to the ASF occurrence in the most affected Member States (MSs) of the European Union (EU), the methodology of the present study focused on the evaluation of outbreaks and on the dynamics of cases in the targeted Eastern-European states.

\section{Results and Discussion}

Thirteen Eastern-European states reported first occurrences of ASF between 2007 and 2017, as follow: Georgia (date of start of the event: 23/04/2007), Armenia (07/08/2007), The Russian Federation (05/11/2007; first occurrence after 30 years), Azerbaijan (22/01/2008), Ukraine (30/07/2012), Belarus (19/06/2013), Lithuania (24/01/2014), Poland (13/02/2014), Latvia (25/06/2014), Estonia (02/09/2014), Moldova (17/09/2016), the Czech Republic (21/06/2017), and Romania (27/07/2017) (Ramishvili, 2007; Baghiyan, 2007; Nepoklonov, 2007; Murshud, 2008; Zhuk, 2012; Pivavarchyk, 2013; Milius, 2014; Jazdzewski, 2014; Balodis, 2014; Partel, 2014; Stamati, 2016; Semerád, 2017; Grigore, 2017).

The source of the outbreak or the origin of infection in the first notifications of ASF emergence were unknown or inconclusive for nine states: Georgia, Armenia, Ukraine, Estonia, Latvia, Lithuania, Poland, the Czech Republic and Romania (Ramishvili, 2007; Baghiyan, 2007; Zhuk, 2012; Milius, 2014; Jazdzewski, 2014; Balodis, 2014; Partel, 2014; Semerad, 2017; Grigore, 2017). In Azerbaijan, Belarus and Moldova immediate notification of the ASF emergence, pointed towards the sources of the outbreaks as fomites (humans, vehicles, feed, etc.) or the practice of swill feeding (Murshud, 2008; Pivavarchyk, 2013; Stamati, 2016). Only the Russian Federation considered that the transboundary migration of wild boars across the subalpine grassland, along the Argoun and the Shatoy-Argoun rivers, led to the re-emergence of ASF in their territory (Nepoklonov, 2007). However, the main route of ASFv introduction, indicated by the Member States of the European Union, was the transboundary circulation of boars, even if the mechanisms of the virus spreading in European wild boars are not obvious (Sanchez-Cordon et al, 2018). Three cycles of ASFv transmission have been identified and described: the domestic cycle, the sylvatic cycle and the tick-pig cycle. However, the spread of ASFv by both, wild and domestic pigs, and also the one relating to human alimentary customs/traditions shouldn't be ignored.

Although the main characteristics of ASF are the same, dominant transmission cycles in African and European outbreaks have been shown to be different, including the substantial involvement of wild boars in the Eastern European area (Sanchez-Cordon et al, 2018).

The analysis of the new outbreak dynamics shows an increase in the cross-border spread of ASFv in the last years, mainly after 2014, with a maximum of eight states in 2017 (Table 1). This trend was demonstrated in the increase in number of new outbreaks: 92.55\% (5268/5692) in 2014-2017 (Table 2). 
CBU INTERNATIONAL CONFERENCE ON INNOVATIONS IN SCIENCE AND EDUCATION

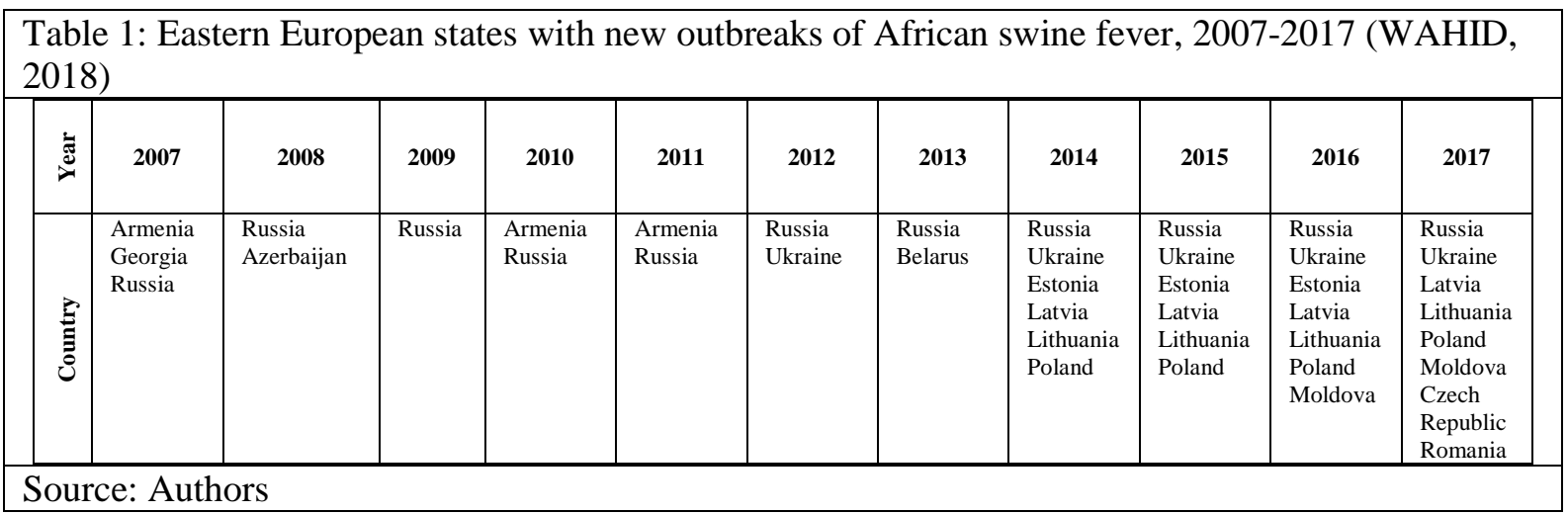

Table 2: New outbreaks of African swine fever in Eastern Europe, 2007-2017 (WAHID, 2018)

\begin{tabular}{|l|c|c|c|c|c|c|c|c|c|c|c|}
\hline Country & $\mathbf{2 0 0 7}$ & $\mathbf{2 0 0 8}$ & $\mathbf{2 0 0 9}$ & $\mathbf{2 0 1 0}$ & $\mathbf{2 0 1 1}$ & $\mathbf{2 0 1 2}$ & $\mathbf{2 0 1 3}$ & $\mathbf{2 0 1 4}$ & $\mathbf{2 0 1 5}$ & $\mathbf{2 0 1 6}$ & $\mathbf{2 0 1 7}$ \\
\hline Armenia & 13 & 0 & 0 & 4 & 11 & 0 & 0 & 0 & 0 & 0 & 0 \\
\hline Georgia & 60 & 0 & 0 & 0 & 0 & 0 & 0 & 0 & 0 & 0 & 0 \\
\hline The Russian Federation & 1 & 45 & 54 & 78 & 54 & 31 & 69 & 75 & 81 & 291 & 188 \\
\hline Azerbaijan & 0 & 1 & 0 & 0 & 0 & 0 & 0 & 0 & 0 & 0 & 0 \\
\hline Ukraine & 0 & 0 & 0 & 0 & 0 & 1 & 0 & 11 & 19 & 84 & 152 \\
\hline Belarus & 0 & 0 & 0 & 0 & 0 & 0 & 2 & 0 & 0 & 0 & 0 \\
\hline Estonia & 0 & 0 & 0 & 0 & 0 & 0 & 0 & 7 & 718 & 327 & 0 \\
\hline Latvia & 0 & 0 & 0 & 0 & 0 & 0 & 0 & 172 & 762 & 245 & 91 \\
\hline Lithuania & 0 & 0 & 0 & 0 & 0 & 0 & 0 & 48 & 96 & 323 & 330 \\
\hline Poland & 0 & 0 & 0 & 0 & 0 & 0 & 0 & 32 & 54 & 104 & 852 \\
\hline Moldova & 0 & 0 & 0 & 0 & 0 & 0 & 0 & 0 & 0 & 2 & 7 \\
\hline Czech Republic & 0 & 0 & 0 & 0 & 0 & 0 & 0 & 0 & 0 & 0 & 193 \\
\hline Romania & 0 & 0 & 0 & 0 & 0 & 0 & 0 & 0 & 0 & 0 & 4 \\
\hline Total & $\mathbf{7 4}$ & $\mathbf{4 6}$ & $\mathbf{5 4}$ & $\mathbf{8 2}$ & $\mathbf{6 5}$ & $\mathbf{3 2}$ & $\mathbf{7 1}$ & $\mathbf{3 4 5}$ & $\mathbf{1 7 3 0}$ & $\mathbf{1 3 7 6}$ & $\mathbf{1 8 1 7}$ \\
\hline
\end{tabular}

Source: Authors

The limited information provided by Estonia, Latvia, Lithuania and Poland, EFSA et al (2017) did not allow us to perform a feasible spatial-temporal analysis (BYM) for considering potential risk factors that could be linked to the presence of ASF cases in a given region (EFSA et al, 2017). However, while the domestic swine in the Russian Federation and their contaminated products still pose a major risk for new ASF outbreaks (Kolbasov et al, 2017), in MS the main actor seems to be the wild boar. In only four years (2014-2017), Estonia, Latvia, Lithuania, Poland and the Czech Republic notified 92.28\% (4042/4380) of all ASF outbreaks reported in Eastern Europe between 2007 and 2017 (Table 3).

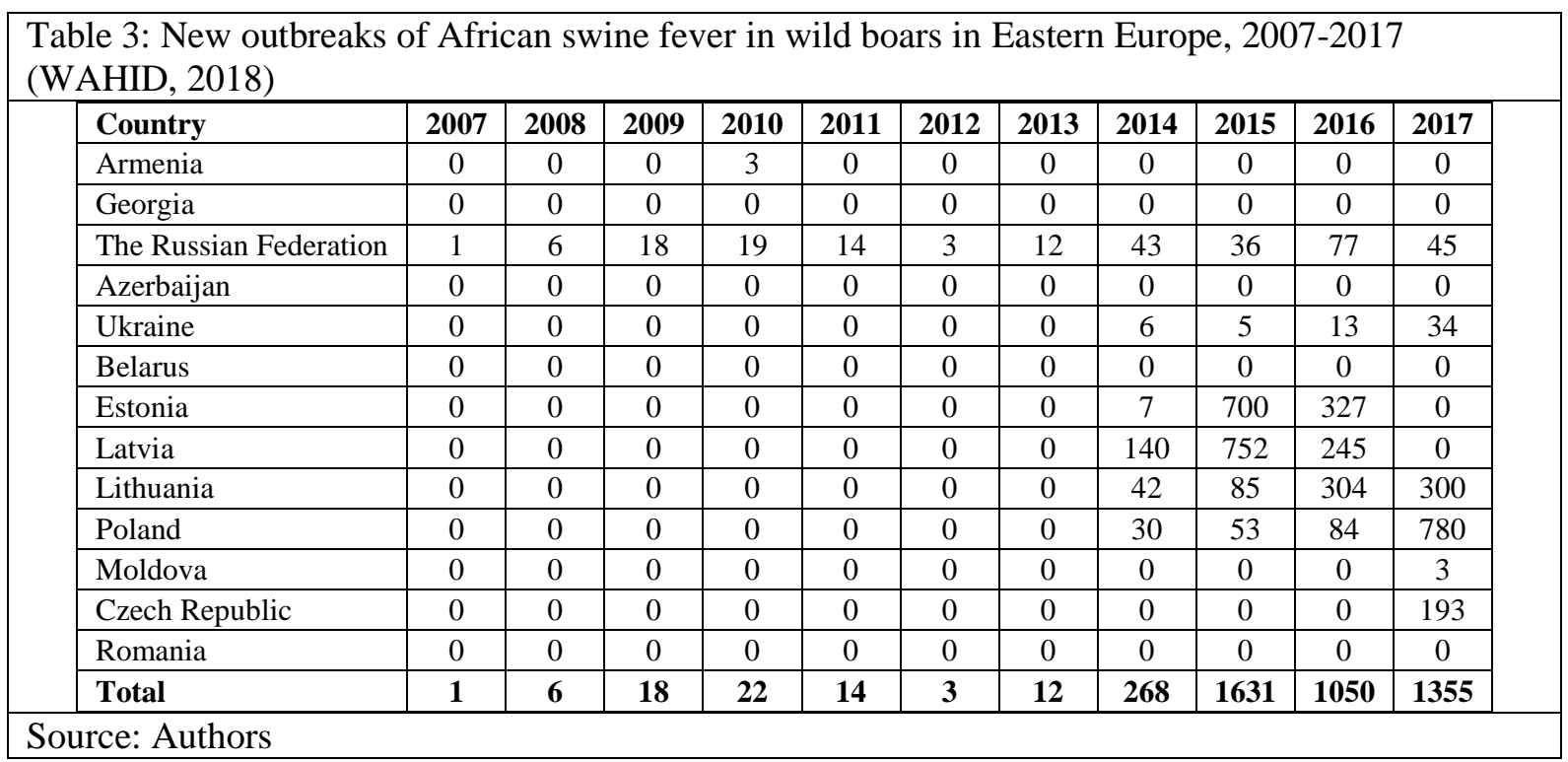


Although the disease is spreading relatively slowly in wild boars, in the EU affected countries during 2017, Poland and the Czech Republic reported almost all ASF outbreaks due to wild boars (Table 2 and 3): $91.55 \%$ (780/852) in Poland and 100\% (193/193) in the Czech Republic. Even more, in the Czech Republic the ASF disease was reported for the first time, while in Poland ASF outbreaks in wild boars have grown (EFSA et al, 2017).

A stacked area chart of ASF outbreaks, by country. It shows the magnitude of each country's outbreak to the total value across the ASF outbreak trends, 2007-2017. This magnitude can also be seen in Figures $1-4$.

Figure 1: A stacked area chart of ASF outbreaks, by country. It shows the magnitude of each country's outbreak to the total value across the ASF outbreaks trend, 2007-2017

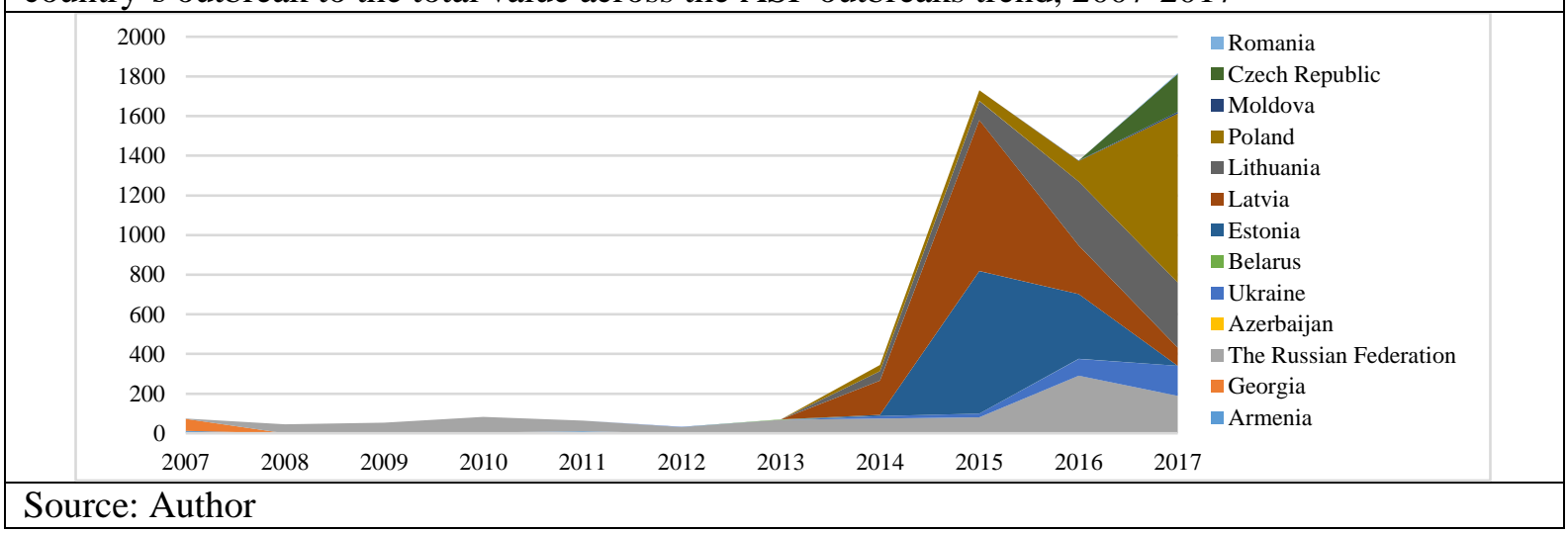

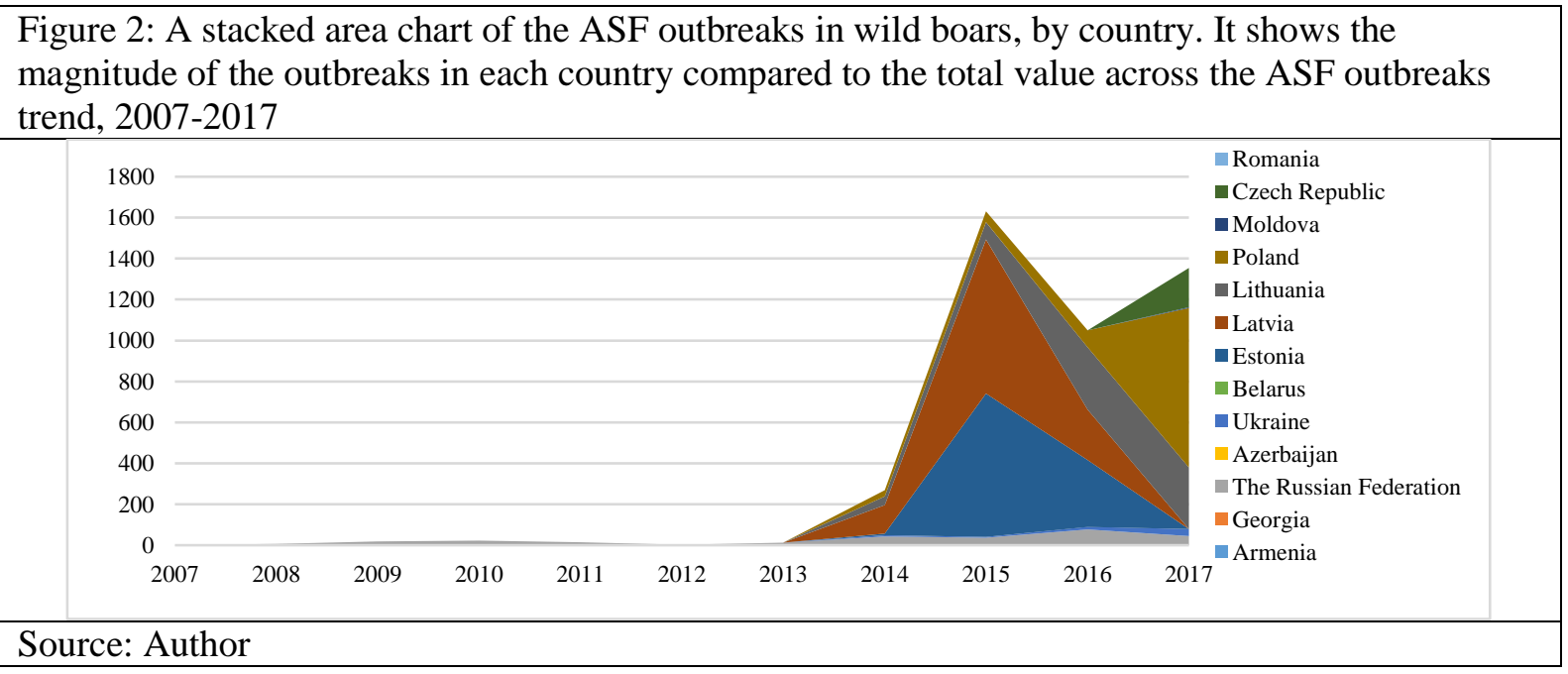

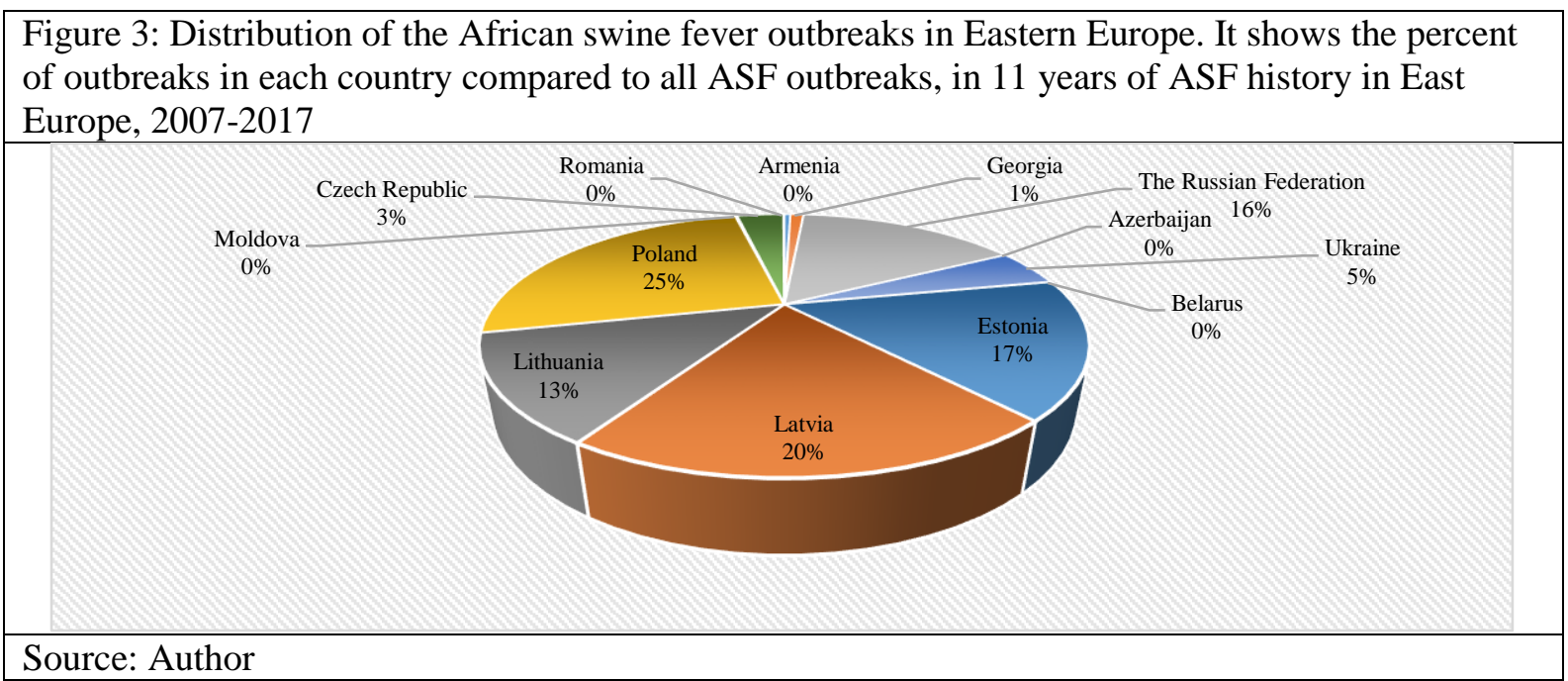




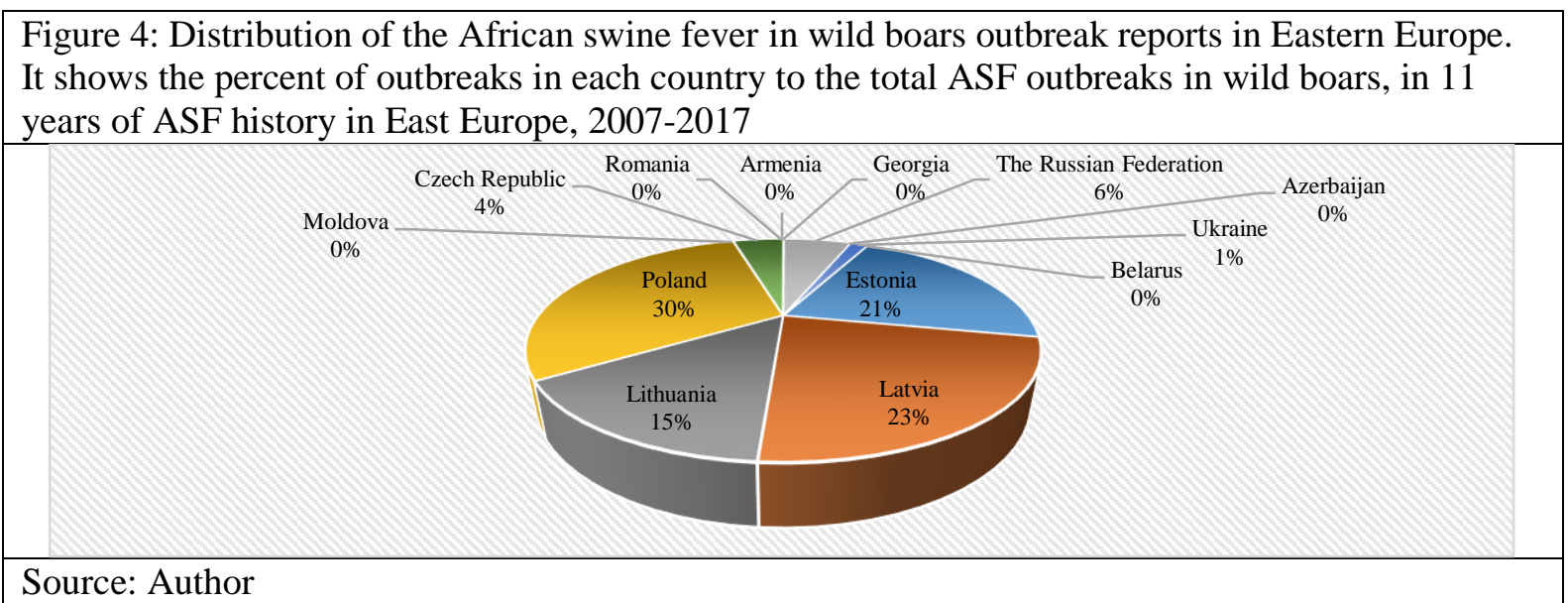

Concerning the dynamic of ASF cases and mortality, in 2007-2011 a different evolution of indicators was noticed between pigs and wild boars (Figures 5-8), with a progressive reduction in cases in pigs and an increase in cases in wild boars, especially in the last four years. Also, the general trend of ASF cases increased slowly in last four years (Figures 9 and 10).

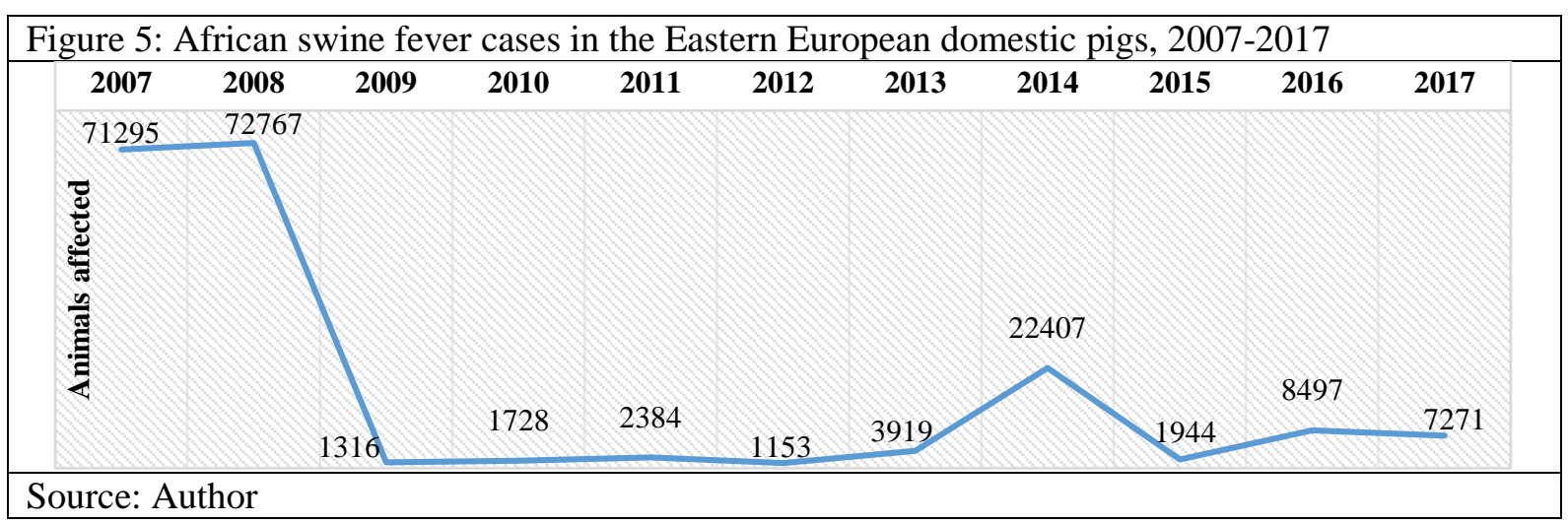

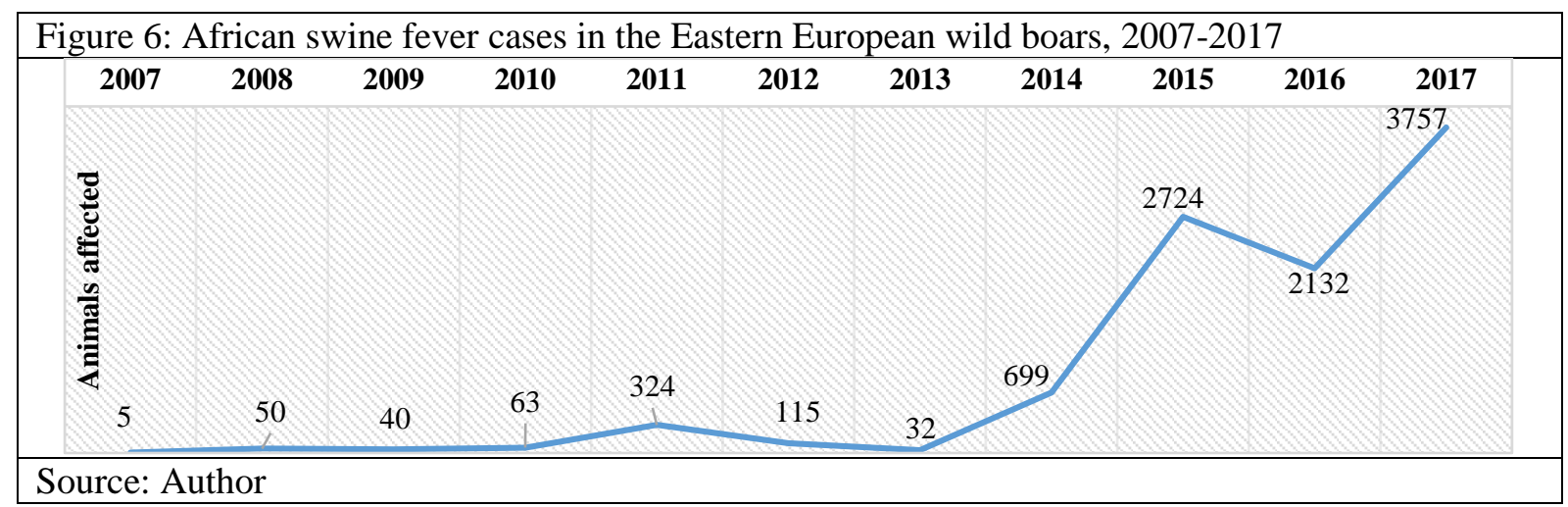

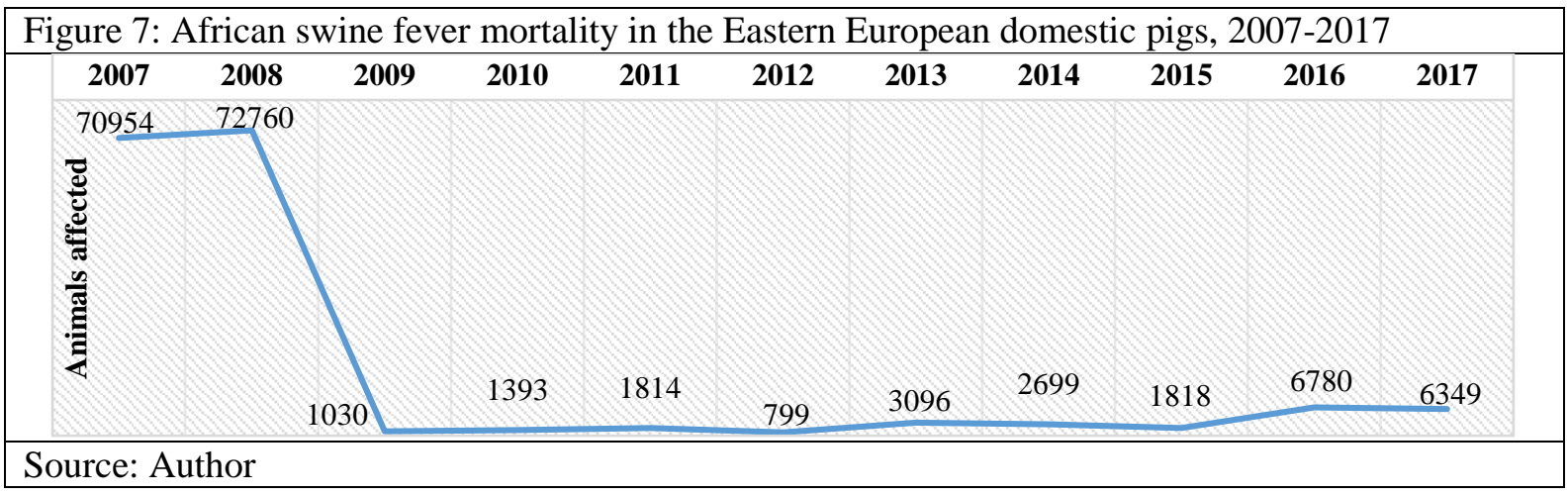




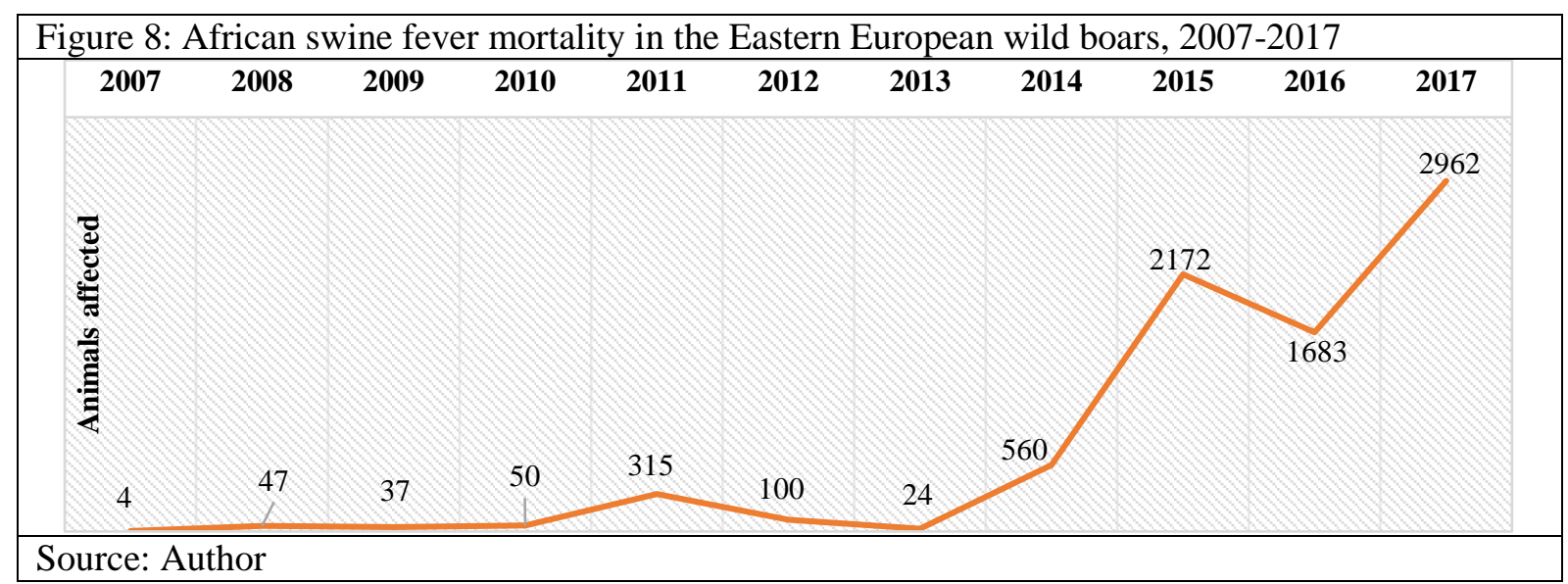

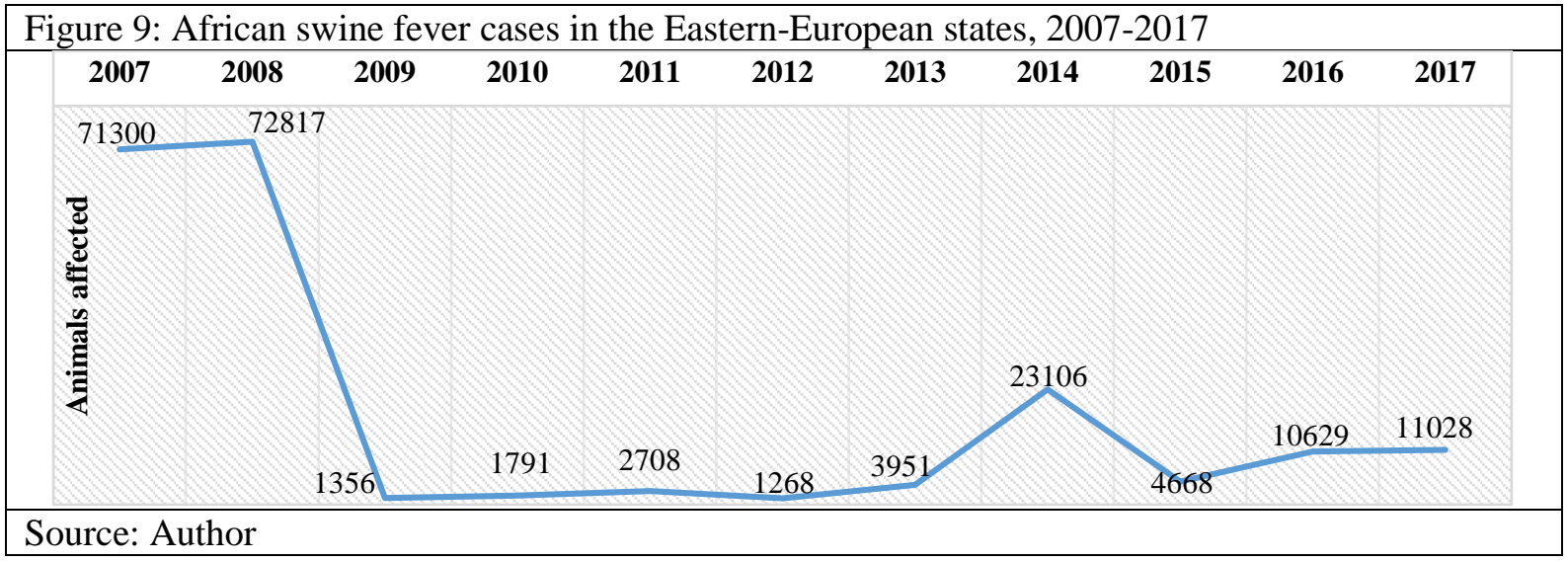

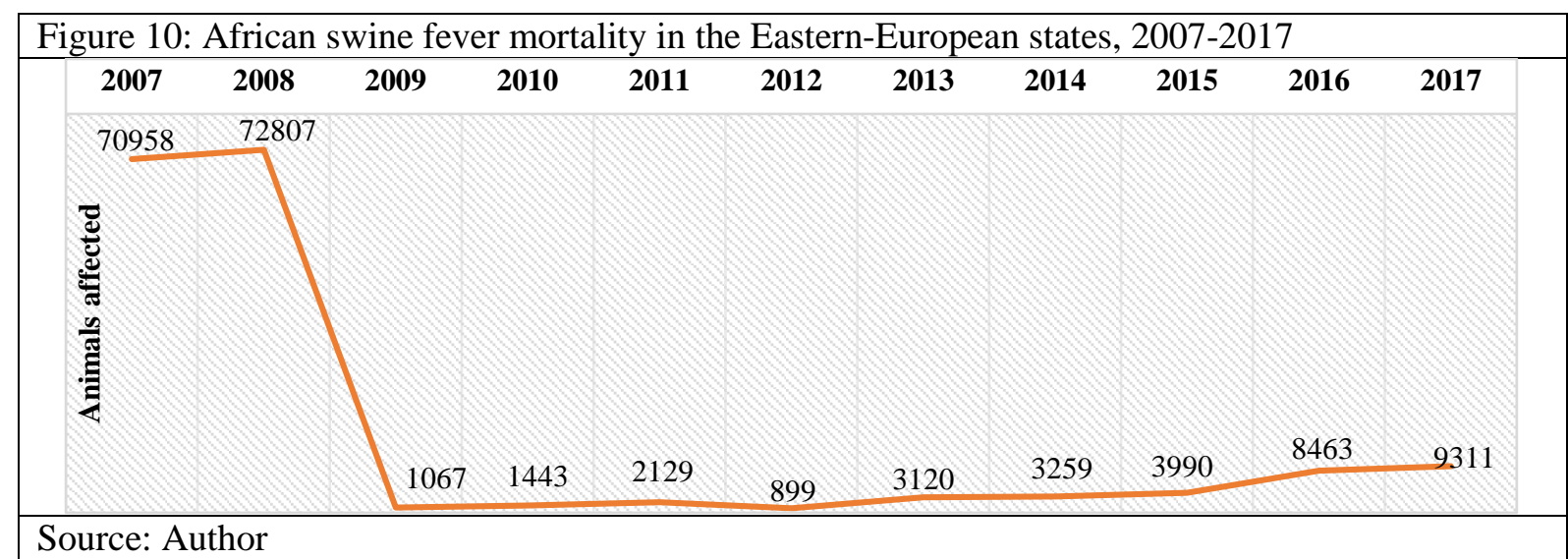

As Sanchez-Cordon et al. (2018) points out, ASFv isolates are still highly virulent and the mortality of affected animals, both domestic pigs and wild boar, is great in Eastern Europe and the Russian Federation. However, the survivor animals should be considered in affected areas to design effective control and eradication plans (Mur et al, 2016). Also, considering that the main mechanisms of virus spread in European are not clear (Sanchez-Cordon et al, 2018), then all ways of ASFv dissemination should be evaluated in each new outbreak.

\section{Conclusion}

The increased spread of ASFv in Eastern Europe supports the potential risk of spread in other European regions and the maintaining of high vigilance mainly for the cross-border spread of ASF. None of the ways to disseminate ASFv should be excluded, and the origin of the first outbreaks remains unknown or inconclusive in Eastern EU states. 


\section{References}

Baghiyan, G. (2007). African swine fever, Armenia. World Animal Health Information Database [WAHID]. Retrieved from http://www.oie.int/wahis_2/public/wahid.php/Reviewreport/Review? page_refer=MapEventSummary\&reportid=6051

Balodis, M. (2014). African swine fever, Latvia. World Animal Health Information Database [WAHID]. Retrieved from http://www.oie.int/wahis_2/public/wahid.php/Reviewreport/Review? page_refer=MapEventSummary\&reportid=15485

Beltran-Alcrudo, D., Lubroth, J., Depner, K. \& De La Rocque, S. (2008). African swine fever in the Caucasus. EMPRES Watch. Rome, FAO, 1-8. Retrieved from ftp://ftp.fao.org/docrep/fao/011/aj214e/ aj214e00.pdf.

European Food Safety Authority [EFSA], Cortinas Abrahantes, J., Gogin, A., Richardson, J., \& Gervelmeyer, A. (2017). Scientific report on epidemiological analyses on African swine fever in the Baltic countries and Poland. EFSA Journal, 15(3):4732, 73 pp. DOI:10.2903/j.efsa.2017.4732

Gallardo, M. C., Reoyo, A. de la T., Fernandez-Pinero, J., Iglesias, I., Munoz, M. J., \& Arias, M. L. (2015). African swine fever: a global view of the current challenge. Porcine Health Management, 1, 21. DOI:10.1186/s40813-015-0013-y

Grigore, M. (2017). African swine fever, Romania. World Animal Health Information Database [WAHID]. Retrieved from http://www.oie.int/wahis_2/public/wahid.php/Reviewreport/Review? reportid=24456

Jazdzewski, K. (2014). African swine fever, Poland. World Animal Health Information Database [WAHID]. Retrieved from http://www.oie.int/wahis_2/public/wahid.php/Reviewreport/Review? page_refer=MapEventSummary\&reportid=14793

Kolbasov, D., Titov, I., Tsybanov, S., Gogin, A., \& Malogolovkin, A. (2018). African Swine Fever Virus, Siberia, Russia, 2017. Emerging Infectious Diseases, 24(4), 796-798. DOI:10.3201/eid2404.171238

Milius, J. (2014). African swine fever, Lithuania. World Animal Health Information Database [WAHID]. Retrieved from http://www.oie.int/wahis_2/public/wahid.php/Reviewreport/Review? page_refer=MapEventSummary\&reportid=14690 Montgomery, R.E. (1921). On a form of swine fever occurring in British East Africa (Kenya Colony). J. Comp. Pathol. Ther. 34(3), 159-191. DOI:10.1016/S0368-1742(21)80031-4

Mur, L., Igolkin, A., Varentsova, A., Pershin, A., Remyga, S., Shevchenko, I., Zhukov, I., Sanchez-Vizcaino, J. M. (2016). Detection of African swine fever antibodies in experimental and field samples from the Russian Federation: implications for control. Transboundary and Emerging Diseases, 63(5), e436-e440. DOI:10.1111/tbed.12304

Murshud, I. H. (2008). African swine fever, Azerbaijan. World Animal Health Information Database [WAHID]. Retrieved from http://www.oie.int/wahis_2/public/wahid.php/Reviewreport/Review? reportid=6730

Nepoklonov, E. A. (2007). African swine fever, Russia. World Animal Health Information Database [WAHID]. Retrieved from http://www.oie.int/wahis_2/public/wahid.php/Reviewreport/Review? page_refer=MapEventSummary\&reportid=6546 Partel, A. (2014). African swine fever, Estonia. World Animal Health Information Database [WAHID]. Retrieved from http://www.oie.int/wahis_2/public/wahid.php/Reviewreport/Review? reported=16019

Pivavarchyk, Y. (2013). African swine fever, Belarus. World Animal Health Information Database [WAHID]. Retrieved from http://www.oie.int/wahis_2/public/wahid.php/Reviewreport/Review? page_refer=MapEventSummary\&reportid=13663

Ramishvili, L. (2007). African swine fever, Georgia. World Animal Health Information Database [WAHID]. Retrieved from http://www.oie.int/wahis_2/public/wahid.php/Reviewreport/Review? page_refer=MapFullEventReport\&reportid=5720

Sanchez-Cordon, P. J., Montoya, M., Reis, A. L., \& Dixon, L. K. (2018). African swine fever: A re-emerging viral disease threatening the global pig industry. Veterinary Journal (London, England : 1997), 233, 41-48.

DOI:10.1016/j.tvjl.2017.12.025

Sanchez-Vizcaino, J. M., \& Arias, M. (2012). African swine fever virus, in Diseases of Swine, Tenth Edition. Ed Zimmerman, J. J., Karriker, L. A., Ramirez, A., Schwartz, K. J., \& Gregory W. Stevenson, G. W., Ames, Iowa, SUA, 396404. John Wiley \& Sons

Semerad, Z. (2017). African swine fever, Czech Republic. World Animal Health Information Database [WAHID]. Retrieved from http://www.oie.int/wahis_2/public/wahid.php/Reviewreport/Review? reportid=24159

Stamati, V. (2016). African swine fever, Moldova. World Animal Health Information Database [WAHID]. Retrieved from http://www.oie.int/wahis_2/public/wahid.php/Reviewreport/Review? page_refer=MapEventSummary\&reportid=21095

World Animal Health Information Database [WAHID] (2018). WAHIS Interface.

http://www.oie.int/wahis_2/public/wahid.php/Diseaseinformation/Immsummary/listoutbreak

Zhuk, A. (2012). African swine fever, Ukraine. World Animal Health Information Database [WAHID]. Retrieved from http://www.oie.int/wahis_2/public/wahid.php/Reviewreport/Review? page_refer=MapEventSummary\&reportid=12168 